\title{
LXA4 protects against blue-light induced retinal degeneration in human A2E-laden RPE cells and Balb-c mice
}

\author{
Tianhua Xie ${ }^{1,2}$, Jiping $\mathrm{Cai}^{2}$, Yong $\mathrm{Yao}^{2}$, Chao Sun ${ }^{2}$, Qian Yang ${ }^{2}$, Meili Wu ${ }^{3}$, Zifan Xu ${ }^{2}$ Xiaodong Sun ${ }^{1}$, \\ Xiaolu Wang ${ }^{3}$
}

${ }^{1}$ Department of Ophthalmology, Shanghai General Hospital of Nanjing Medical University, Shanghai, China; ${ }^{2}$ Department of Ophthalmology, The Affiliated Wuxi People's Hospital of Nanjing Medical University, Wuxi, China; ${ }^{3}$ Center of Clinical Research, The Affiliated Wuxi People's Hospital of Nanjing Medical University, Wuxi, China

Contributions: (I) Conception and design: X Wang, X Sun, T Xie; (II) Administrative support: None; (III) Provision of study materials or patients: None; (IV) Collection and assembly of data: T Xie, C Sun, Q Yang, M Wu, Z Xu; (V) Data analysis and interpretation: X Wang, X Sun, J Cai, Y Yao; (VI) Manuscript writing: X Wang, T Xie; (VII) Final approval of manuscript: All authors.

Correspondence to: Xiaodong Sun. Department of Ophthalmology, Shanghai General Hospital of Nanjing Medical University, Shanghai 200080, China. Email: xdsun@sjtu.edu.cn; Xiaolu Wang. Center of Clinical Research, The Affiliated Wuxi People’s Hospital of Nanjing Medical University, 299 Qingyang Road, Wuxi 214023, China. Email: xlwang@njmu.edu.cn.

Background: Age-related macular degeneration (AMD) is one of the leading causes of permanent visual impairment in the elderly. Blue light (BL) has been reported to cause retinal damage and contribute to the onset and development of severe AMD. N-retinylidene-N-retinylethanolamine (A2E), a lipofuscin fluorophore, accumulates with ageing in the retinal pigment epithelium (RPE) cells. Once exposed to BL, A2E easily oxidizes to A2E-epoxides, causing oxidative-stress injury to the retina. Lipoxin A4 (LXA4), an endogenous anti-antioxidant lipid, plays a key role in multiple organs by binding to the formyl-peptide receptor-like 1 (FPRL1). This study examined the protective effects of LXA4 on oxidative-stress injury induced by BL exposure, and clarified the underlying mechanisms in cultured RPE cells and Balb-c mice.

Methods: LXA4 diluent was orally administered to mice before retinal degeneration was established. Optical coherence tomography, retinal histology, and RPE cell injury were assessed.

Results: LXA4 administration significantly ameliorated retinal damage as evidenced by the thicknesses of the retinal layers and the tight junctions of RPE cells in vivo. LXA4 inhibited BL-induced reactive oxygen species (ROS) production, reduced tight junctions, and the death of A2E-laden RPE cells. LXA4 also potently increased the expression of haem oxygenase-1 (HO1) and $\mathrm{NAD}(\mathrm{P}) \mathrm{H}$ quinone oxidoreductase 1 (NQO1), probably by decreasing the association between nuclear factor erythroid 2-related factor 2 (NRF2) and Kelch-like ECH (Epichlorohydrin) -associated protein 1 (Keap1), and ameliorating NRF2 nuclear translocation and the antioxidant response element (ARE) deoxyribonucleic acid (DNA) binding activity.

Conclusions: Our results showed that LXA4 ameliorated retinal degeneration, and should be considered in the prevention and treatment of AMD.

Keywords: Age-related macular degeneration (AMD); retinal degeneration; lipoxin A4 (LXA4); blue light; oxidative stress

Submitted Jun 08, 2021. Accepted for publication Aug 05, 2021.

doi: $10.21037 / \mathrm{atm}-21-3390$

View this article at: https://dx.doi.org/10.21037/atm-21-3390 


\section{Introduction}

Aging is a multifactorial process and a major risk factor for age-related diseases, such as cancer, type 2 diabetes mellitus, cardiovascular diseases, and ocular diseases. Age-related macular degeneration (AMD) is a progressive disease closely related to visual damage and blindness in the elderly population (1). Non-exudative AMD is characterized by sub-retinal pigment epithelium (RPE) drusen deposits and the geographic atrophy of the RPE, while exudative AMD is characterized by choroidal neovascularization (2). The underlying mechanisms of AMD need to be determined to provide effective treatment.

RPE cells phagocytose the photoreceptor membrane discs, containing rhodopsin, lipids, and phosphatidyl pyridinium bisretinoid over a lifetime (3). Lipidsoluble yellow-brown pigments (lipofuscin) are byproducts produced by the lysosomal degradation process. Accumulated lipofuscin mediates the disruption of cellular activities, such as anti-oxidation reactions, metabolic processes, and lysosomal degradative functions (4). $\mathrm{N}$-retinylidene-N-retinylethanolamine (A2E), a by-product generated from all-trans-retinal and ethanolamine, is one of the major fluorophores of RPE lipofuscin. Many in vitro studies have employed A2E-laden RPE cells to examine the pathogenesis of macular degeneration (5). The blue-light (BL) illumination of A2E-laden RPE cells causes reactive oxygen species (ROS)-induced apoptosis, inflammation, and complement (system) activation. In vivo, BL exposure induces the elimination of ROS, the accumulation of lipofuscin, local inflammation, apoptosis, and ultimately, the degeneration of photoreceptors $(6,7)$.

RPE cells are equipped with antioxidants that maintain the pro-oxidant/antioxidant balance (8). The nuclear factor erythroid 2-related factor 2 (NRF2) involved in the antioxidant system controls the coordinated expression of the antioxidant response element (ARE)-regulated genes of downstream detoxifying enzymes, such as $\mathrm{NAD}(\mathrm{P}) \mathrm{H}$ quinone oxidoreductase 1 (NQO1) and haem oxygenase-1 (HO1) by combining with the promoter (9). Under resting conditions, NRF2 and Kelch-like ECH-associated protein 1 (Keap1) in the RPE cytoplasm bind to each other, and the antioxidant genes are not stimulated (10). When ROS production increases, NRF2 separates from the Keap1 protein, enters the nucleus and activates the expression of HO1, NQO1 and other antioxidant enzymes, thus playing an antioxidant role (11). The materials constitute a pleiotropic cellular defense, which eliminates ROS and xenobiotics, detoxifies electrophiles, and maintains the intracellular reducing potential to attenuate BL-induced inflammation and oxidative damage, all of which could represent promising strategies for the prevention of AMD (12).

Lipoxins are the first members of endogenous 'braking signals' of inflammation in vivo (13). Lipoxin A4 (LXA4) is one of the most critical lipoxins. It plays an effective antiinflammatory and antioxidant role in several organs by binding to formyl-peptide receptor-like 1 (FPRL1, also referred to as FPR2 or ALXR) (13). Additionally, LXA4 may exert biological effects through other mechanisms; for example, the neuroprotective effects of LXA4 are partially regulated by peroxisome proliferator-activated receptor gamma in rodent stroke (14). Aspirin-triggered LXA4 (ATL, 15-epi-LXA4), a LXA4 epimer from arachidonic acid, also plays a key endogenous anti-inflammation role via the LXA4 receptor, FPRL1 (15). However, there are few reports on the signaling involved in BL-induced retinal damage.

We sought to determine the role of LXA4 or Aspirin/ FPRL1 signaling in oxidative stress-induced RPE cell injury and retinal degeneration in mice. The signaling of the NRF2-HO1 axis was also investigated. Our novel findings in relation to the effect of LXA4 on BL-induced retinal degeneration suggest that LXA4 could potentially serve as a therapeutic approach for AMD. We present the following article in accordance with the ARRIVE reporting checklist (available at https://dx.doi.org/10.21037/atm-21-3390).

\section{Methods}

\section{Animals}

Male Balb-c mice were kept at the animal center of the Affiliated Wuxi People's Hospital of Nanjing Medical University under standard conditions. The mice were ventilated on corresponding days after being anaesthetized with a ketamine $(120 \mathrm{mg} / \mathrm{kg}$, i.p.) and xylazine $(6 \mathrm{mg} / \mathrm{kg}$, i.p.) mixture. After the adequacy of the anesthesia was established, the eyes were obtained for further analyses. All animal experimental procedures in the present study were approved and monitored by the Institutional Animal Care and Use Committee of the Affiliated Wuxi People's Hospital of Nanjing Medical University. Animal care and method procedures were carried out in accordance with the National Institute's Health Guide for the Care and Use of Laboratory Animals (National Academy of Sciences Press, 2011).

For the BL exposure model, 5-week-old mice were 
acclimated in dark cages and orally administered LXA4 or Aspirin every $24 \mathrm{~h}$ for $3 \mathrm{~d}$. Next, the mice were orally administered LXA4 or Aspirin and exposed to BL (10,000 lux, $430 \mathrm{~nm}$ ) for $1 \mathrm{~h}$. LXA4 or Aspirin was dissolved in 100\% ethanol (v/v) and mixed with the food. After the ethanol was completely volatilized, the food was fed to the mice daily. Control mice were fed the volatilized ethanol-treated food daily. BL exposures and treatments were conducted every $24 \mathrm{~h}$ for $14 \mathrm{~d}$. After the last exposure, the mice were placed in a dark environment for an extra $24 \mathrm{~h}$.

\section{Cells}

ARPE-19 cells (ATCC, Manassas, VA, USA) was $>90 \%$ positive for acetylated low-density lipoprotein, and cultured in Dulbecco's modified Eagle's medium (DMEM) containing fetal bovine serum (10\%) and antimycotics and antibiotics (1\%). Cells were treated with $20 \mu \mathrm{M} \mathrm{A2E}$ for $6 \mathrm{~h}$ and washed with phosphate-buffered saline, the supernatant was removed and replaced with DMEM without phenol red (31053028, Life Technologies, CA, USA). LXA4 (90410, Cayman Chemical Company, Ann Arbor, MI, USA) was added to cells at the designated concentration for $30 \mathrm{~min}$. All the cells, except the control cells, were subject to BL illumination (1,000 lux, $430 \mathrm{~nm}$ ) for the indicated time.

\section{Retinal imaging}

The optical coherence tomography (OCT) system (Micron IV; Phoenix Research Labs, Pleasanton, CA, USA) was used to take live-fundus images.

\section{Hematoxylin and eosin staining}

The eyes were obtained and fixed in paraformaldehyde (4\%) overnight. The retina and the RPE-choroid-sclera complexes were dehydrated and embedded. 5 - $\mu$ m-thick sections were taken for hematoxylin and eosin (H\&E) staining, and images were collected under an Olympus BX51 light microscope (Olympus, Tokyo, Japan).

\section{Immunofluorescence analysis}

An immunofluorescence analysis of zonula occludens-1 (ZO-1) and FPRL1 was conducted, followed by incubated with secondary antibodies (1:500; Thermo Fisher Scientific, CA, USA) in RPE cells or tissue sections, as previously described (16). For the whole-mount analysis, flat-mounts were fixed in $4 \%$ buffered neutral formalin fixatives (Biosharp, Beijing, China) for $2 \mathrm{~h}$. The muscle, optic nerve, and connective tissue were removed from the back of the eye, and the lens and cornea were also removed. The connection of the retina to the optic nerve was severed. 4 radial incisions were made in the RPE flat mounts, which were further fixed for $1 \mathrm{~h}$ at room temperature. The flatmounts were blocked with $10 \%$ goat serum for $1 \mathrm{~h}$. They were then incubated with ZO-1 (1/500, ab216880, Abcam) overnight at $4{ }^{\circ} \mathrm{C}$. After a secondary antibody (1/500, Alexa Fluor 488 goat anti-rabbit IgG, Thermo Fisher Scientific) and Hoechst 33342 (1:1,000; Thermo Fisher Scientific) were used, images were taken using a confocal microscope (Leica, Heidelberg, Germany).

\section{Detection of ROS levels}

Cells were incubated with dihydroethidium (DHE; Life Technologies, Carlsbad, CA, USA) for $1 \mathrm{~h}$. Images were taken under a microscope.

\section{WST-1 assay}

The cell proliferation reagent, water soluble tetrazolium-1 (WST-1) was employed to determine the viability as described previously (17). Briefly, the cultured cells $\left(5 \times 10^{4}\right)$ were incubated with WST for $2 \mathrm{~h}$ at $37{ }^{\circ} \mathrm{C} .450-\mathrm{nm}$ absorbance was detected.

\section{Flow cytometry}

Apoptosis was evaluated by annexin V-propidium iodide (PI) staining (\#640914; BioLegend, San Diego, CA, USA). Cells were suspended in $400 \mu \mathrm{L}$ binding buffer and incubated with annexin V-FITC (fluoresceine isothiocyanate) $(5 \mu \mathrm{L})$ and PI $(10 \mu \mathrm{L})$ in the dark. A Cytomics FC500 instrument (Beckman Coulter, Miami, FL, USA) was used to evaluate the rates of cell apoptosis.

\section{Transfection of siRNA}

Three independent small inhibitory ribonucleic acid (siRNA) sequences of NRF2 (siRNA\#1 \#3) were obtained from Ribobio (Guangzhou, China) and employed to inhibit NRF2 expression. Cells were transfected with siNRF2 or a small-interference non-genomic sequence (siNC) at a concentration of $20 \mathrm{nM}$ according to the instructions of the commercial kit (Thermo Fisher Scientific, CA, USA). 


\section{Immunoprecipitation and western blot analysis}

Cells were lysed and immunoprecipitated with anti-NRF2 antibody (Cell Signaling Technology). $30 \mu \mathrm{L}$ protein A/ $\mathrm{G}$ agarose beads (GE Healthcare, Pittsburgh, PA, USA) were used for $4 \mathrm{~h}$ to detect the association between NRF2 and Keap1.Isolated protein was subjected to sodium dodecyl sulfate polyacrylamide gel electrophoresis and transferred to polyvinylidene-fluoride membranes (Millipore, Billerica, MA, USA). The primary antibodies included HO1 (Abcam, 1/1,000, ab68477), NQO1 (Abcam, 1/1,000, ab28947), NRF2 (Novus, 1/1,000, NBP132822), FPRL1 (Novus, 1/1,000, NLS1878) and Keap1 (Santa Cruz Biotechnology, 1/200, sc-514914). Secondary antibodies (ZSGB-BIO, 1/3,000, ZB-2301 and ZSGBBIO, 1/3,000, ZB-2305) were also employed. Equal protein loading among the samples was confirmed by the $\beta$-actin antibody (Sigma-Aldrich, St. Louis, MO, USA) and the Lamin B1 antibody (Abcam, Cambridge, MA, USA). An enhanced chemiluminescence system (West Pico Kit, Pierce, Loughborough, UK) was employed to detect the membrane signals. The relative intensities were analyzed with Image $\mathrm{J}$ software.

\section{EMSA}

The binding activity analysis of ARE employed in this study has been described previously (18). An electrophoretic mobility shift assay (EMSA) kit (Thermo Fisher Scientific, Waltham, MA, USA) was used. A biotin end-labelled deoxyribonucleic-acid (DNA) duplex of sequences containing the ARE binding site (5'-ACT GAG GGT GAC TCA GCA AAA TC-3', 3'-TGA CTC CCA CTG AGT CGT TTT AG-5') was employed.

\section{RNA quantification}

RNA was detected by reverse transcription polymerase chain reaction (19). The primer sequences are described in Table S1.

\section{Statistical analysis}

Data are reported as the mean \pm standard error of the mean (SEM). An analysis of variance was conducted to compare the multiple groups using the GraphPad Prism 5 statistic software (Prism v5.0; GraphPad Software, La Jolla, CA, USA). A P $<0.05$ was considered statistically significant.

\section{Results}

LXA4 treatment prevents BL-induced retina degeneration in vivo

We employed the widely used BL animal model in Balb-c mice, which display symptoms of RPE dystrophy and atrophic AMD (20). Previous studies have reported that BL exposure significantly induces oxidative and nitrative stress, as evidenced by increased mRNA levels of NADPH oxidase subunits p22phox, p47phox, and gp91phox (21). In the present study, gp91phox, p22phox, and p47phox mRNA were significantly increased in the RPE-choroid-sclera complexes of mice in the BL-exposed group (see Figure S1). It has been reported that LXA4 exerts its anti-inflammatory and antioxidant effects by binding to FPRL1 (22). Thus, we examined the expression of FPRL1 in vivo, and found that FPRL1 immunolocalization overlapped with the RPE layer in the BL-induced retina injury (see Figure S2).

In the animal model of retinal degenerative changes caused by BL, the retinas were examined by spectral domain optical coherence tomography after 10,000-lux bright light exposure. Retinal damage was found in mice exposed to $\mathrm{BL}$, and the most prominent degeneration occurred in the outer nuclear layer (ONL). The structures of the ONL, the outer plexiform layer (OPL), and the photoreceptor layer were poorly delineated $14 \mathrm{~d}$ after BL exposure (see Figure 1A). As the H\&E-stained images show, the RPE cells showed continuous lining and were arranged in an orderly manner in the untreated control retinas. However, the RPE cell nuclei were hyper-chromatic after BL exposure. The thicknesses of the abovementioned layers were notably lower in the BL-exposed mice than the control mice. However, the administration of LXA4 protected the retinal layers against illumination-induced degeneration, especially the ONL. No significant improvement was noted in volatilized ethanol-treated BL illumination mice compared with the untreated BL illumination mice (see Figure $1 B-1 D$ ). To further examine whether LXA4 exerted a protective role via the FPRL1 receptor in BL-exposed mice, we compared the effects of LXA4 with Aspirin, which initiates the biosynthesis of the Aspirin-triggered 15-epiLXA4. Aspirin may also block BL-induced changes in the thicknesses of the ONL (see Figure 1B,1C).

\section{LXA4 treatment inbibits BL-induced RPE cell death}

Methylene blue staining and whole-mount RPE analyses were employed to examine the BL-illuminated changes of 
A

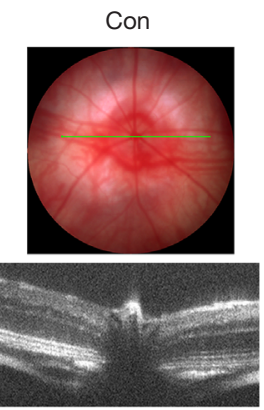

B

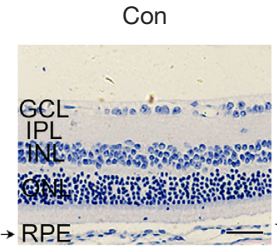

C

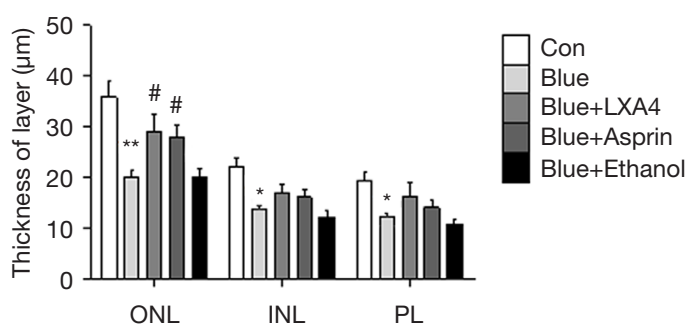

Blue

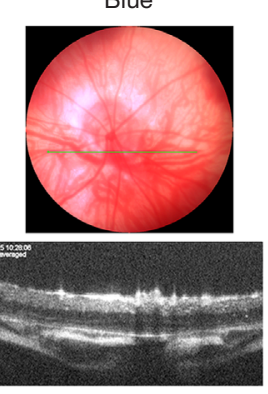

Blue

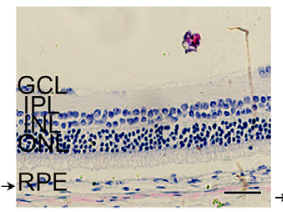
$\rightarrow$ RPE:
Blue+LXA4

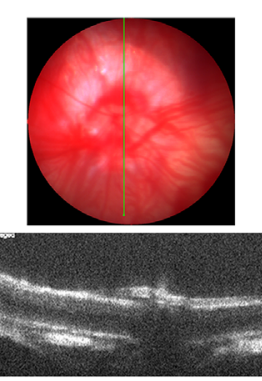

Blue+LXA4

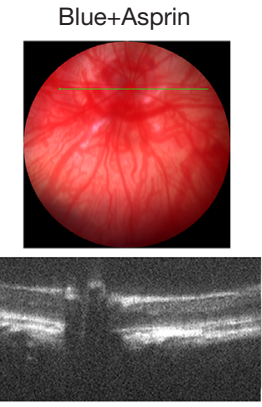

Blue+Asprin

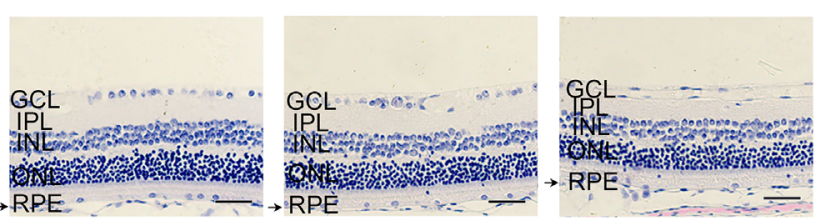

Blue+Ethanol

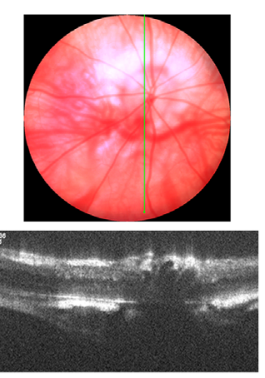

Blue+Ethanol

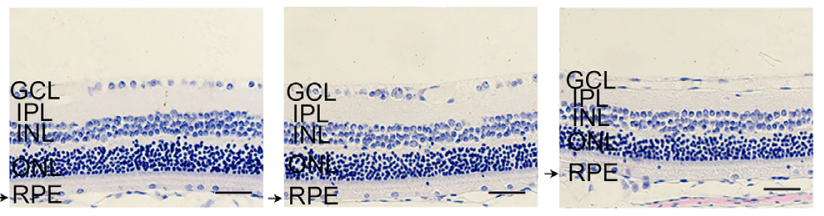

D

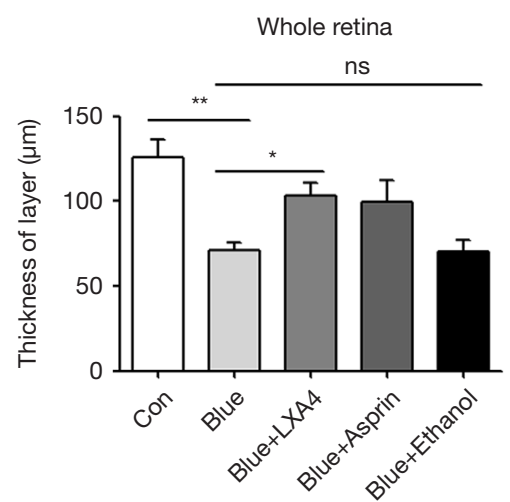

Figure 1 LXA4 prevents morphological changes in BL-illuminated eyes. (A) Images of color fundus and OCT $14 \mathrm{~d}$ after BL exposure. (B) $\mathrm{H} \& \mathrm{E}$ staining of the retina and RPE-choroid-sclera complexes $14 \mathrm{~d}$ after BL illumination; scale bar, $25 \mu \mathrm{m}$. The arrow indicates the RPE layer. (C,D) The thickness of the retina was quantified by H\&E staining. The Blue + Ethanol group was treated with volatilized ethanol control before BL exposure. The results are expressed as the mean $\pm \mathrm{SEM} ; \mathrm{N}=6,{ }^{*} \mathrm{P}<0.05$ and ${ }^{* *} \mathrm{P}<0.01$ : Blue vs. control; ${ }^{*} \mathrm{P}<0.05$ : Blue + LXA4 or Blue + Asprin vs. Blue. GCL, ganglion cell layer; INL, inner nuclear layer; IPL, inner plexiform layer; ONL, outer nuclear layer; RPE, retinal pigment epithelial (/epithelium) layer.

RPE cells. Patchy RPE degeneration was observed on day 14 post BL exposure; however, LXA4 or Aspirin treatment compensated for the BL illumination changes (see Figure $2 A$ ). Tight junctions were visualized by immunostaining using an antibody against ZO-1. BL illumination led to a decreased number of ZO-1 positive cells, indicating bloodretina barrier damage. However, LXA4 or Aspirin treatment repressed that effect (see Figure 2B,2C).

\section{BL exposure markedly increases oxidative stress damage in A2E-laden RPE cells in vitro}

DHE staining showed that treating A2E-laden RPE cells
(A2E group) with BL illumination at different time points significantly increased the ROS content, especially at $15 \mathrm{~h}$ (A2E + B15). Conversely, group simply exposed to BL for $1 \mathrm{~h}(\mathrm{~A} 2 \mathrm{E}+\mathrm{B} 1)$, and group exposed to BL for $1 \mathrm{~h}$ followed by returning to the normal environment for $14 \mathrm{~h}$ (A2E + B1R14) showed only a slight increase in the intracellular ROS level (see Figure $3 A, 3 B$ ). To confirm photo-oxidative damage in the RPE cells, we performed a Cell Counting Kit-8 (CCK8) assay for cell viability at various times after BL illumination. BL exposure significantly induced A2Eladen RPE cell injury at $15 \mathrm{~h}$ (see Figure 3C). Additionally, the tight-junction properties of RPE were visualized by ZO-1 staining. A significant loss of barrier function after 
A

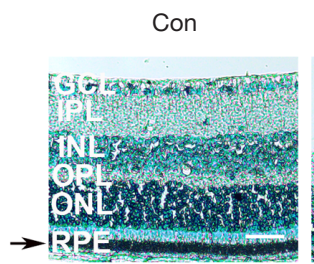

B

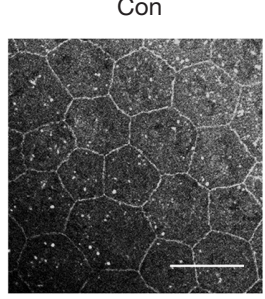

Blue

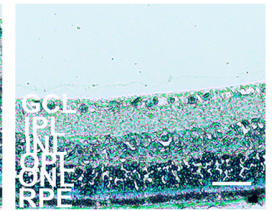

Blue

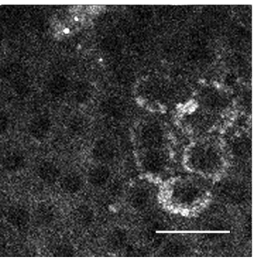

Blue+LXA4

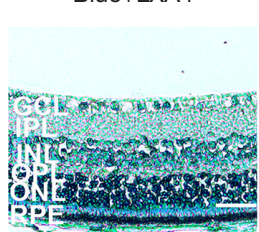

Blue+LXA4

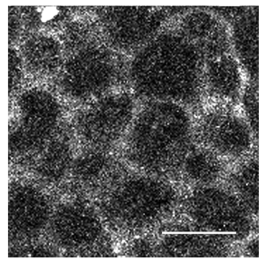

Blue+Asprin

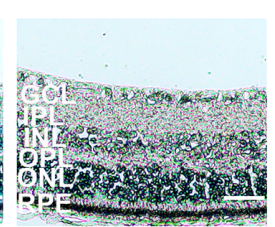

Blue+Asprin

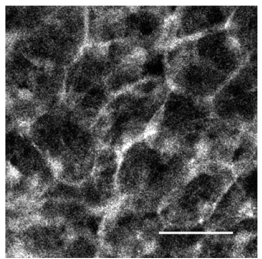

Blue+Ethanol

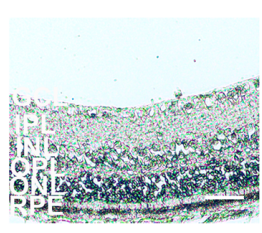

Blue+Ethanol

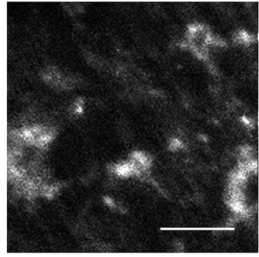

C

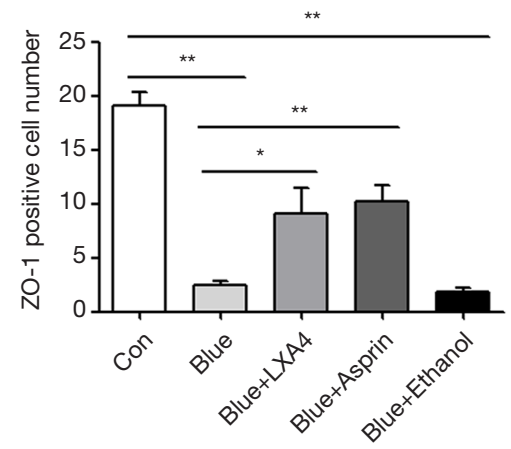

Figure 2 LXA4 abolishes BL-associated RPE damage. (A) Methylene blue staining of cryo-sections revealed the details of damaged RPE cells in Balb-c mice; scale bar, 25 m. (B) Tight junctions visualized by ZO-1 staining. After $14 \mathrm{~d}$ of BL illumination, there was a reduction in the tight junctions; scale bar, $25 \mu \mathrm{m}$. (C) Statistical analysis of ZO-1-positive cells. The results are expressed as the mean $\pm \mathrm{SEM} ; \mathrm{N}=6$, ${ }^{*} \mathrm{P}<0.05$ and ${ }^{* *} \mathrm{P}<0.01$. RPE, retinal pigment epithelial (/epithelium) layer.

BL exposure for $15 \mathrm{~h}$ was observed (see Figure 3D,3E).

\section{LXA4 prevents photic damage in RPE cells}

The flow cytometry analysis showed that BL exposure significantly increased the rate of apoptotic cells; however, treatment with LXA4 (50 and $100 \mathrm{nM}$ ) prevented BL illumination-induced apoptosis of A2E-laden RPE cells (see Figure $4 A, 4 B$ ). The DHE staining results showed that compared to the vehicle control group, a 5.6-fold $(\mathrm{n}=4$, $\mathrm{P}<0.01)$ increase in ROS production was observed in the BL-exposed A2E-laden RPE cells. Further, a 0.6-fold $(\mathrm{n}=4, \mathrm{P}<0.01)$ reduction in ROS production was observed in the LXA4 (100-nM)-treated cells versus untreated BLexposed A2E-laden RPE cells (see Figure 4C,4D). This was consistent with the ZO-1 staining results for the RPE cells (see Figure 4E,4F).

\section{BL illumination increases NRF2/HO1 pathway activation in RPE cells}

NRF2-HO1 signaling has been shown to inhibit oxidative stress injury (23). Thus, we examined the effects of $\mathrm{BL}$ illumination on signaling pathway activation. BL illumination led to some elevation of HO1 and NQO1 protein levels and HO1 mRNA expression at $15 \mathrm{~h}$ in A2Eladen RPE cells (see Figure 5A-5C). In the antioxidant system, NRF2 combines with the DNA promoter to regulate gene expression (9). In our study, the NRF2 translocation from the cytoplasm to the nucleus significantly increased at $15 \mathrm{~h}$ post BL illumination (see Figure 5D-5G).

\section{LXA4 promotes $N R F 2 / H O 1$ patbway activation in $B L-$ exposed RPE cells}

It has been reported that the main regulatory procedures 
A

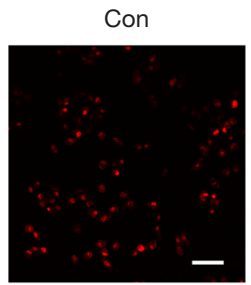

$\mathrm{A} 2 \mathrm{E}+\mathrm{B} 1 \mathrm{R} 14$

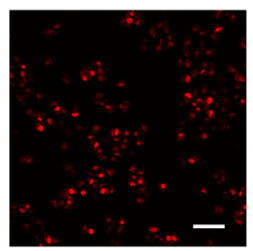

C

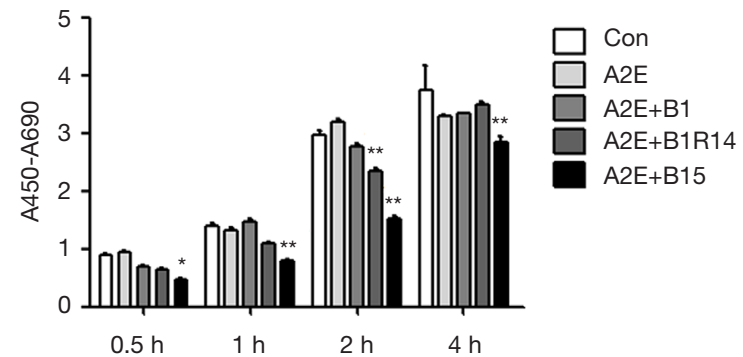

D
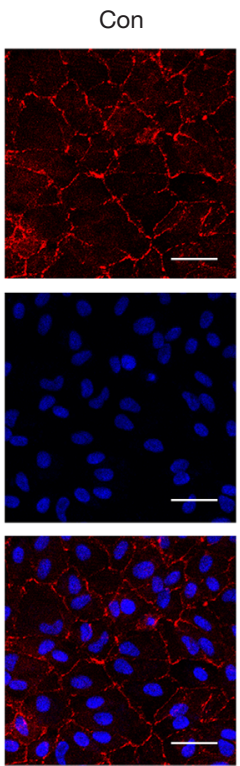

$\mathrm{A} 2 \mathrm{E}$

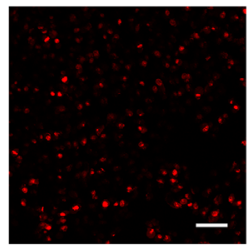

$\mathrm{A} 2 \mathrm{E}+\mathrm{B} 15$
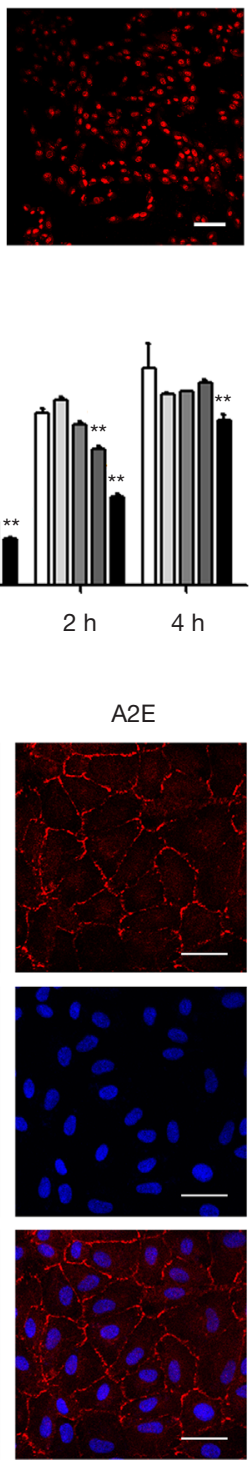

$\mathrm{A} 2 \mathrm{E}+\mathrm{B} 1$
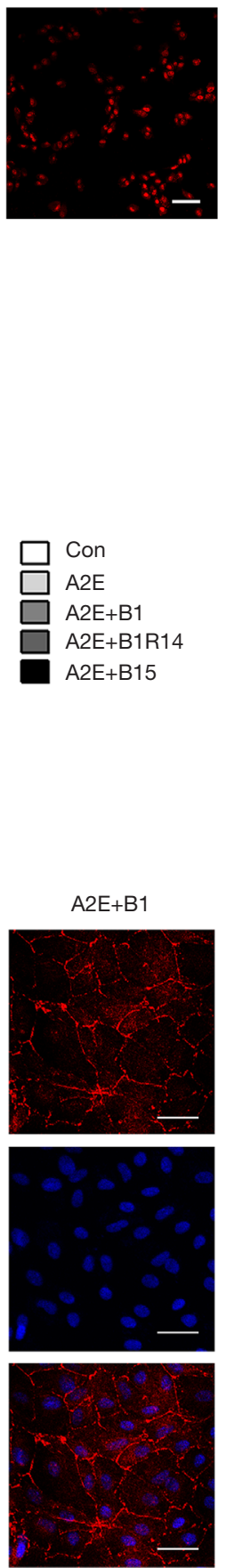

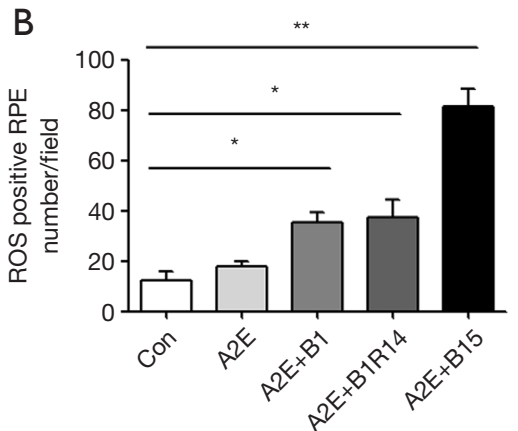

$\mathrm{E}$

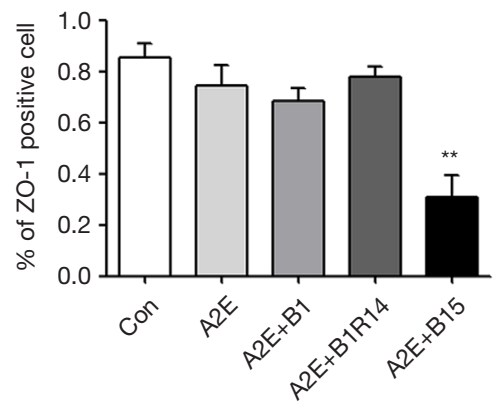

$\mathrm{A} 2 \mathrm{E}+\mathrm{B} 1 \mathrm{R} 14$
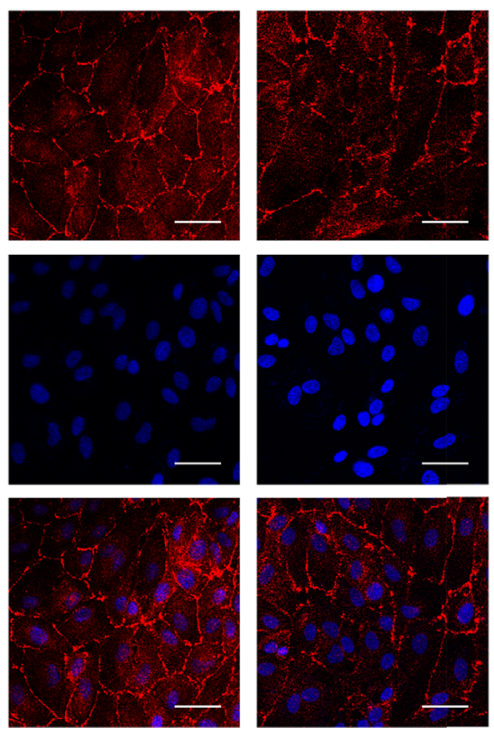

Figure 3 Oxidative stress damage in BL-induced RPE cells. (A) Representative images of DHE staining in the A2E-laden RPE cells at various times post BL illumination; scale bar, $50 \mu \mathrm{m}$. (B) Quantification of DHE-positive cells post BL illumination. (C) Cell viability was detected by CCK8 assay at $0.5,1,2$ and $4 \mathrm{~h}$ after various periods of BL-illumination treatment. (D) Representative images of tight junctions visualized by ZO-1 staining in the RPE cells; scale bar, $25 \mu \mathrm{m}$. (E) Number of ZO-1-positive cells per field. Con, vehicle control; A2E, RPE cells treated with A2E; A2E + B1, RPE cells treated with A2E and exposed to BL for $1 \mathrm{~h}$; A2E + B1R14, RPE cells treated with A2E and exposed to BL for $1 \mathrm{~h}$ and recovered to the normal environment for $14 \mathrm{~h}$; A2E + B15, RPE cells treated with A2E and exposed to BL for $15 \mathrm{~h}$. The results are expressed as the mean $\pm \mathrm{SEM} ; \mathrm{N}=4,{ }^{*} \mathrm{P}<0.05$ and ${ }^{* *} \mathrm{P}<0.01$ : $v$ s. control. RPE, retinal pigment epithelial (/epithelium) layer. 
A

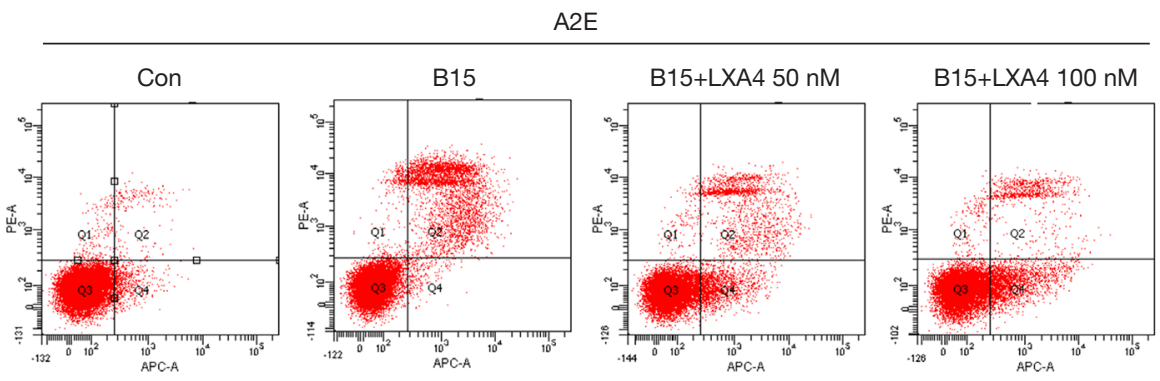

B

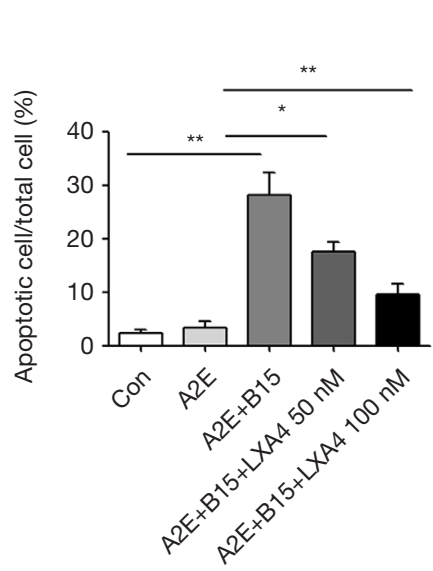

E
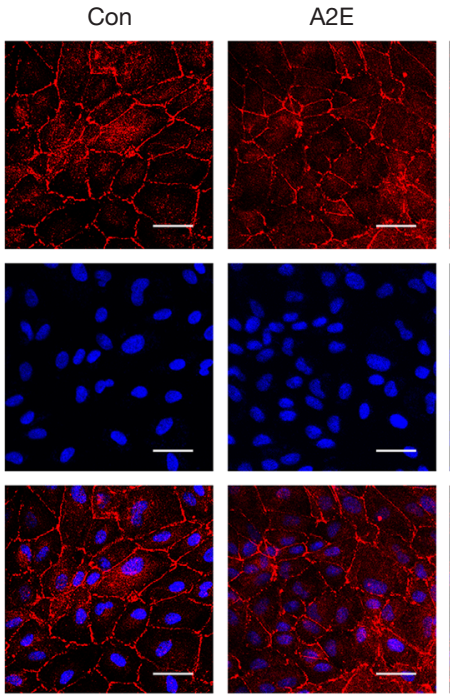

C

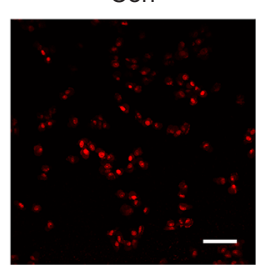

$\mathrm{A} 2 \mathrm{E}+\mathrm{B} 15$

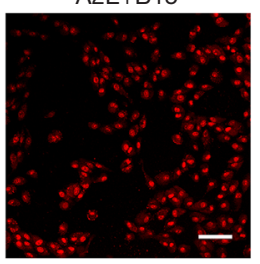

$\mathrm{A} 2 \mathrm{E}+\mathrm{B} 15+\mathrm{LX} 4$

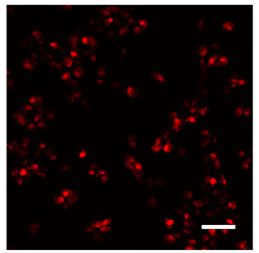

D
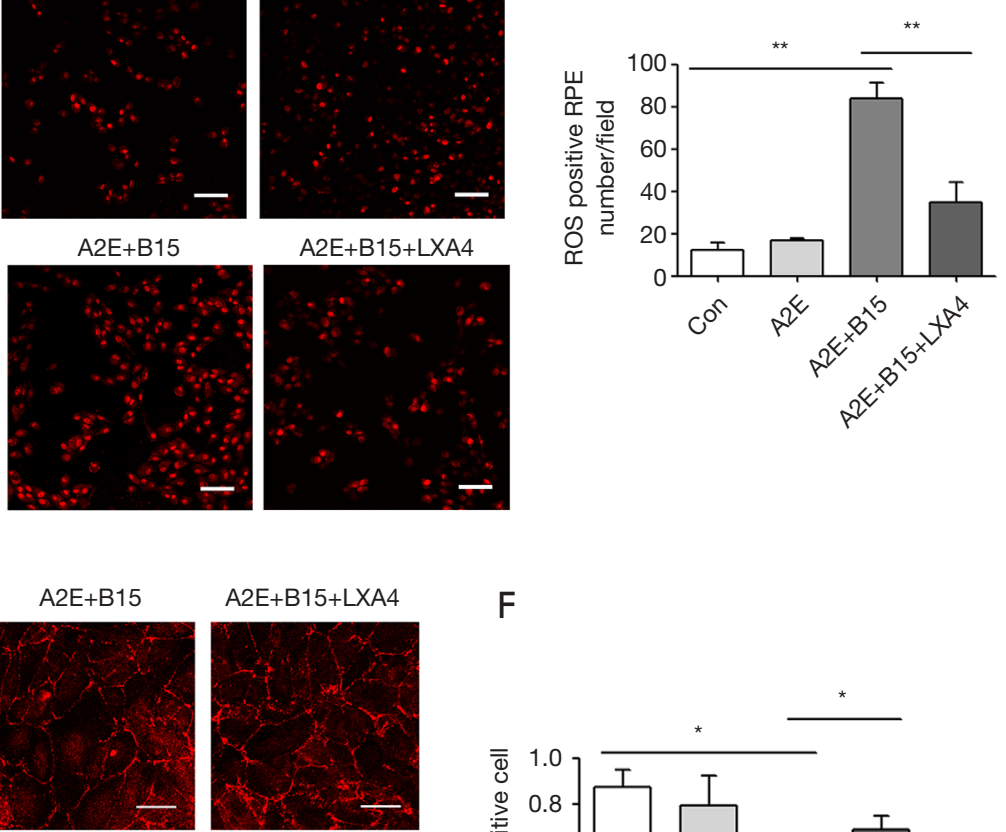

F
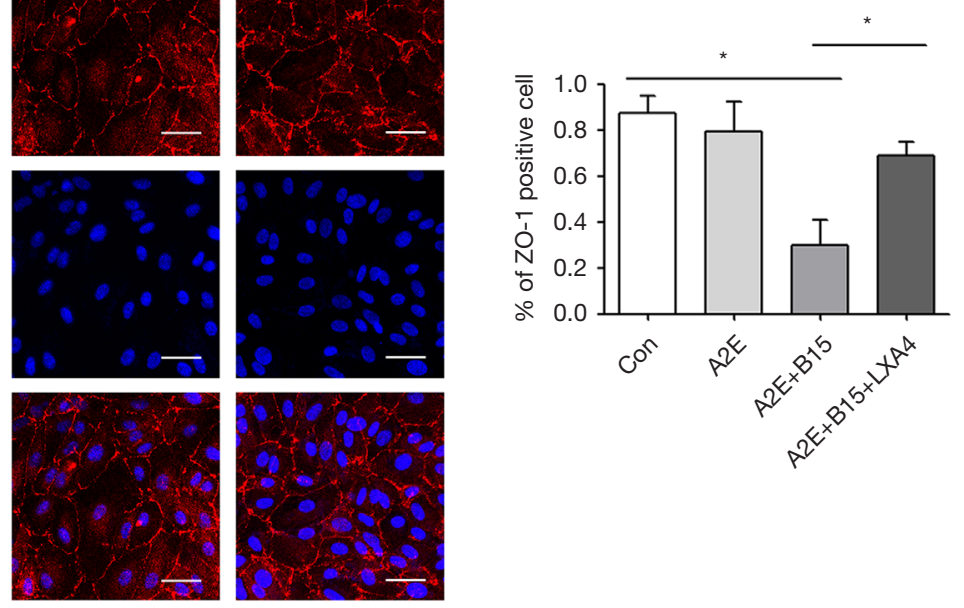

Figure 4 LXA4 abolishes photo-oxidative damage in RPE cells. (A) The BL illumination-induced apoptosis of RPE cells was examined by a flow-cytometry analysis of annexin V-FITC/PI. (B) Quantification of RPE cell apoptosis; N=4. (C) Representative images of DHE staining; scale bar, $50 \mu \mathrm{m}$. (D) Quantification of DHE-positive cells post BL illumination; $\mathrm{N}=4$. (E) Representative images of tight junctions visualized by ZO-1 staining in the RPE cells; scale bar, $25 \mu \mathrm{m}$. (F) Quantification of ZO-1-positive per field. Con, vehicle control; A2E, RPE cells treated with A2E for $6 \mathrm{~h}$; A2E + B15, RPE cells treated with A2E and exposed to BL for 15 h; A2E + B15 + LXA4, RPE cells treated with $\mathrm{A} 2 \mathrm{E}$ and exposed to LXA4 pre-treatment for $30 \mathrm{~min}$ and $\mathrm{BL}$ for $15 \mathrm{~h}$. The results are expressed as the mean $\pm \mathrm{SEM},{ }^{*} \mathrm{P}<0.05$ and ${ }^{* *} \mathrm{P}<0.01$. RPE, retinal pigment epithelial (/epithelium) layer. 
A

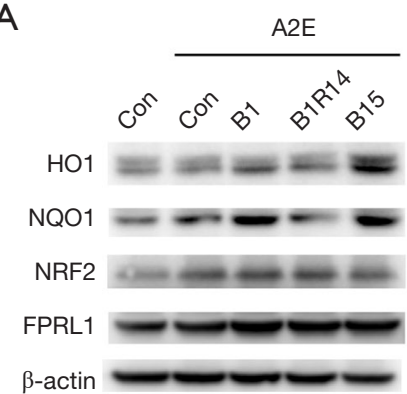

B

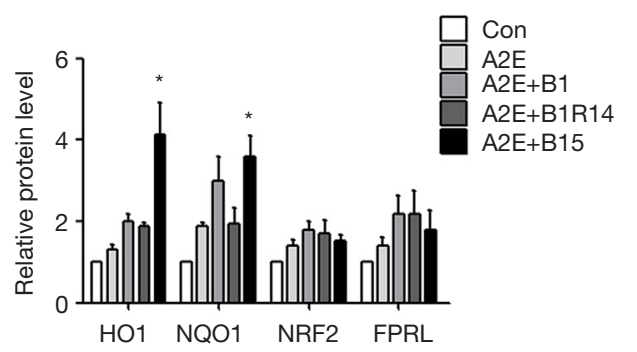

C

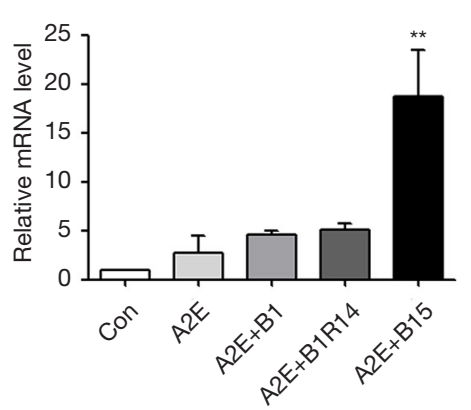

D
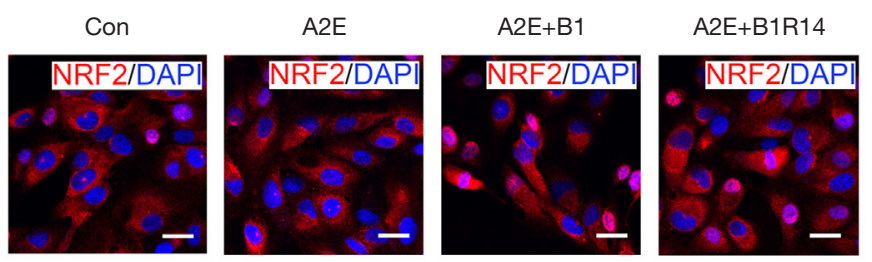

$E$

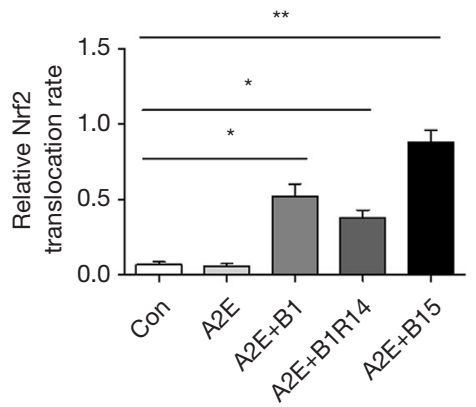

$\mathrm{F}$

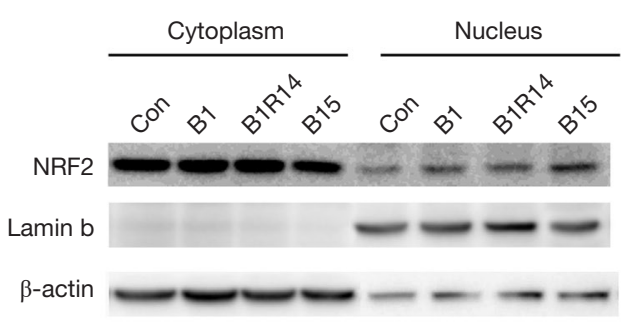

G

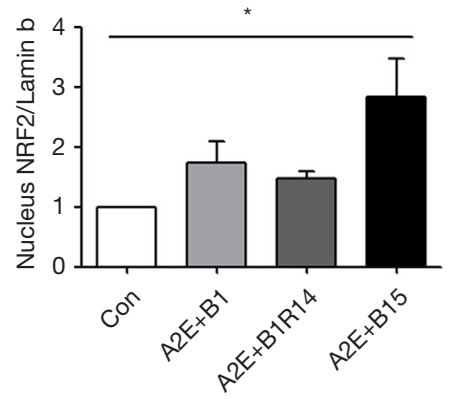

Figure $5 \mathrm{BL}$ induces NRF2/HO1 pathway activation in vitro. (A,B) The protein levels of HO1, NQO1, NRF2, and FPRL1; N=4. (C) The mRNA expression of HO1 in each group's RPE cells; N=4. (D,E) Immunofluorescence assays to evaluate the NRF2 nuclear translocation in each group's RPE cells; scale bar, 25 m; N=4. (F,G) The protein level of nuclear NRF2; N=4. Con, vehicle control; A2E, RPE cells treated with A2E; A2E + B1, RPE cells treated with A2E and exposed to BL for $1 \mathrm{~h}$; A2E + B1R14, RPE cells treated with A2E and exposed to BL for $1 \mathrm{~h}$ and recovered to the normal environment for $14 \mathrm{~h}$; A2E + B15, RPE cells treated with A2E and exposed to BL for $15 \mathrm{~h}$. The results are presented as the mean $\pm \mathrm{SEM},{ }^{*} \mathrm{P}<0.05$ and ${ }^{* *} \mathrm{P}<0.01$ : vs. control. RPE, retinal pigment epithelial (/epithelium) layer.

in the NRF2 activation are the decreased association between the NRF2 and Keap1, which result in increased protein stability and the subsequent nuclear translocation of NRF2 (24). In our study, the protein expression of Keap1 was not significantly changed by BL illumination or LXA4 treatment (see Figure 6A). We also examined the interaction between NRF2 and Keap1 by immunoprecipitation using a specific anti-NRF2 antibody and subsequent immunoblot assay with an anti-Keap1 antibody. BL exposure decreased the association between NRF2 and Keap1, and LXA4 further promoted its separation from Keap1 (see Figure
$6 A, 6 B)$. This was consistent with the expression of HO1 and NQO1 (see Figure 6C,6D). Additionally, BL-exposed RPE cells showed increased NRF2 nuclear translocation and ARE DNA-binding activity, which was heavily exacerbated by LXA4 administration (see Figure 6E-6H).

\section{Blocking the NRF2-HO1 pathway reverses the LXA4 protective effects on BL-induced ROS production}

We transfected siRNA of NRF2 to silence the NRF2 expression. The expression of NRF2 in RPE cells was 
A

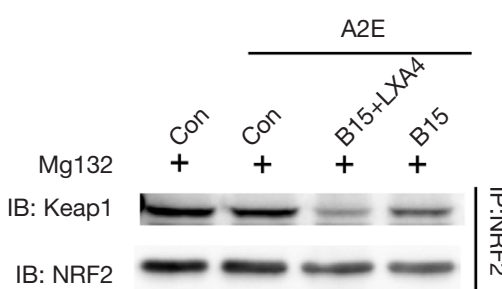

Keap1

NRF2

$\beta$-actin

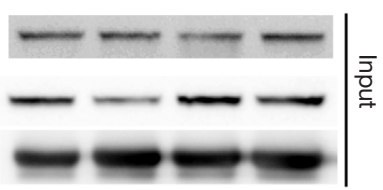

B

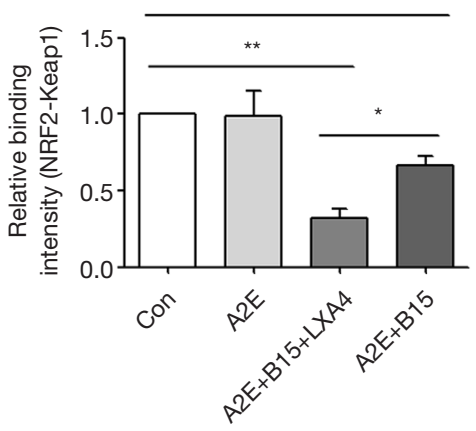

E
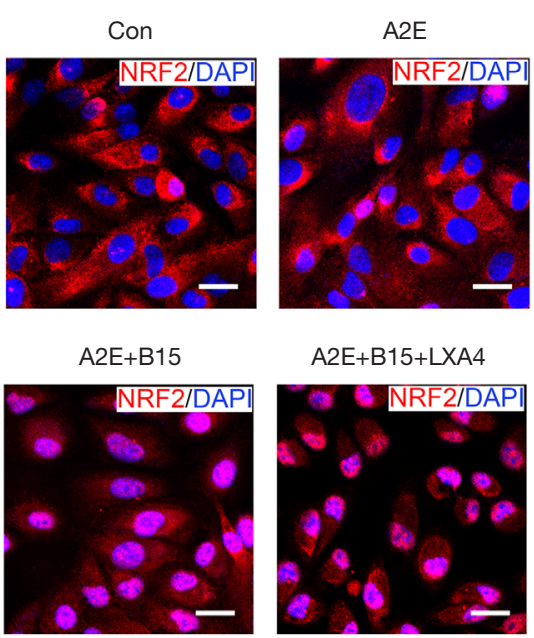

$\mathrm{A} 2 \mathrm{E}+\mathrm{B} 15+\mathrm{LXA} 4$

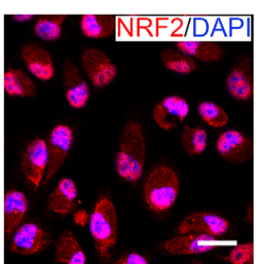

C

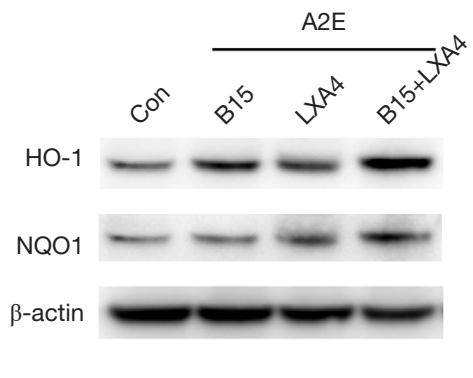

$\mathrm{F}$

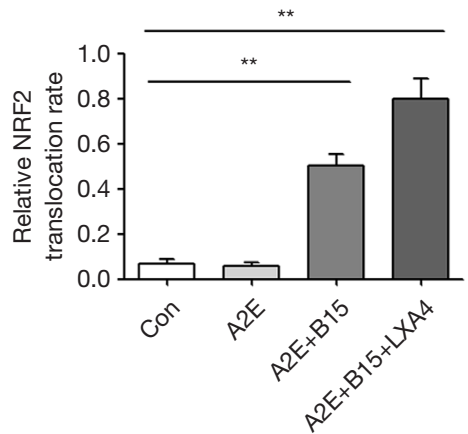

D

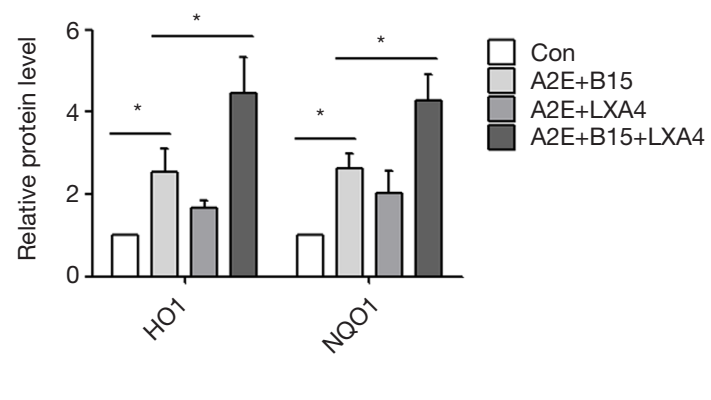

$\mathrm{H}$

G
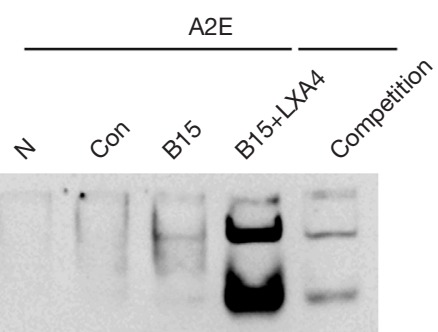

Free probe

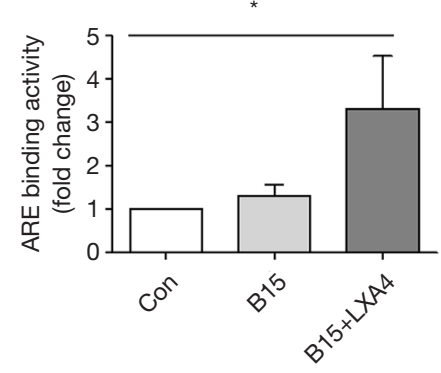

Figure 6 LXA4 further increases the BL-induced activation of NRF2/HO1 pathway. (A,B) Immunoprecipitation of NRF2 and Keap1; N=3. (C,D) The HO1 and NQO1 protein levels are shown; N=4. (E,F) Immunofluorescence assays to evaluate the NRF2 nuclear translocation in each group's RPE cells; scale bar, $25 \mu \mathrm{m} ; \mathrm{N}=4$. (G,H) The binding activity of ARE was examined by EMSA in the nuclear proteins of RPE cells; $\mathrm{N}=3$. Con, vehicle control; A2E, RPE cells treated with A2E; A2E + B15, RPE cells treated with A2E and exposed to BL for 15 h; A2E + B15 + LXA4, RPE cells treated with A2E and exposed to LXA4 pre-treatment for $30 \mathrm{~min}$ and BL for $15 \mathrm{~h}$. The results are presented as the mean $\pm \mathrm{SEM},{ }^{*} \mathrm{P}<0.05$ and ${ }^{* *} \mathrm{P}<0.01$. RPE, retinal pigment epithelial (/epithelium) layer. 
significantly decreased after si-NRF2 transfection (see Figure S3A-S3C). BL-induced increased protein expression of HO1, and NQO1 in the cytoplasm was abrogated following NRF2 silencing (see Figure 7A,7B). Analogously, BL illumination-induced NRF2 nuclear translocation and HO1 mRNA expression were counteracted following NRF2 silencing (see Figure 7C-7E). Further, the cessation of NRF2 counteracted the protective effect of LXA4 on the BL-triggered promotion of ROS production (see Figure $7 F, 7 G)$.

\section{Discussion}

AMD is a progressive chronic disease among individuals over the age of 50 (25). The onset in both eyes can be successive or simultaneous, and it causes severe visual impairment and blindness in many countries (25). Dietary supplements with antioxidants and minerals, such as lutein and zeaxanthin, are commonly recommended as preventive treatments. However, clinical studies have shown that these treatments do not significantly reduce the exudative AMD progression $(26,27)$. Thus, it is crucial to develop novel anti-AMD agents.

BL-induced retinal damage, a classical model of dry $\mathrm{AMD}$, is characterized by $\mathrm{A} 2 \mathrm{E}$ photo-oxidation, and results in the production of ROS (28). BL induces the detachment or degeneration of the retina, and increases inflammatory levels in the retina. Retinal layers thickness (especially ONL) has been shown to decrease as early as $7 \mathrm{~d}$ after BL illumination, and retinal layers have been shown to be markedly degenerated $14 \mathrm{~d}$ post BL illumination (29,30). A2E-laden RPE cells exposed to BL showed a significant decrease in cell viability resulting from the photosensitization of A2E-induced increased ROS generation (31). Consistent with the results of previous studies, we found that fundus, OCT, H\&E and ZO-1 staining showed BL completely disrupted the A2Eladen RPE morphology $14 \mathrm{~d}$ after BL. In patients, RPE degeneration after diabetic retinopathy and RPE tears lead to visual impairment (32). The physiological and morphological damages of RPE are closely involved in visual dysfunction, and the degeneration of RPE cells results in visual acuity reduction in non-exudative AMD (33). We showed that the animal model developed phenotypical and histopathological features consistent with AMD. A young (5-week-old) animal model of retinal degeneration was established. This model is recommended for research on retinal degeneration due to exposure to light, but not for research on lipofuscin accumulation (34). Previous studies have shown that the ARPE-19 cell line is highly sensitive to vascular endothelial growth factor (VEGF), and subsequently induces pigmentation loss and weak tight junctions. This is similar to the pathological characteristics of degeneration in the eye (35). Thus, the ARPE-19 cell line is recommended for RPE function investigations (36). In this study, we employed the ARPE-19 cell line to investigate the effects of LXA4 on the function and pathogenesis of RPE cells.

LXA4 exhibits an anti-inflammatory effect and is involved in many inflammation-associated disease models (37-39). During a previous pre-treatment, attenuated hydrogen peroxide was shown to inhibit cell viability and cell apoptosis (40). However, there is little research on the role of LXA4 in the eye, and no articles appear to have examined its antioxidant effects on RPE cells. LXA4 was orally administered to the mice, and we observed that LXA4 ameliorated the deleterious effects observed in the AMD animal group.

Oxidative stress has been proven to initiate the development of AMD (41). It has been reported that it increases ROS generation, which leads to RPE cell apoptosis (42). Additionally, NRF2/HO1 signaling is a defense mechanism against the oxidative stress of RPE cells (43). The NRF2 is usually activated by endoplasmic reticulum stress or oxidative stress, which induces the upregulation of the level of antioxidant factors, such as NQO1, HO1, CAT, and glutathione. Once ROS is over produced and the redox balance cannot be maintained, the Keap1 translation level is raised. The Keap1 translation leads to the Nrf2 suppression in the cytoplasm (44). Additionally, in RPE cells, intracellular $\mathrm{A} 2 \mathrm{E}$ is easily oxidized by BL illumination and critical to AMD development (12). The increased ROS generation continually consumed glutathione and inhibited the activity of superoxide dismutase, resulting in caspase 3 activation and RPE cell apoptosis (45). This pro-oxidant/ antioxidant balance plays an important role in some chronic inflammatory diseases. In this study, the LXA4 treatment attenuated BL exposure-induced ROS generation and inhibited RPE cell apoptosis. Further, the LXA4 treatment inhibited the combination of NRF2 and Keap1, promoted NRF2 nuclear translocation, and subsequently increased the gene expression of NRF2 and its target genes (i.e., $\mathrm{HO} 1$ and NQO1).

In this study, we showed that LXA4 plays an important role in preventing the oxidant damage of RPE cells in a 
A

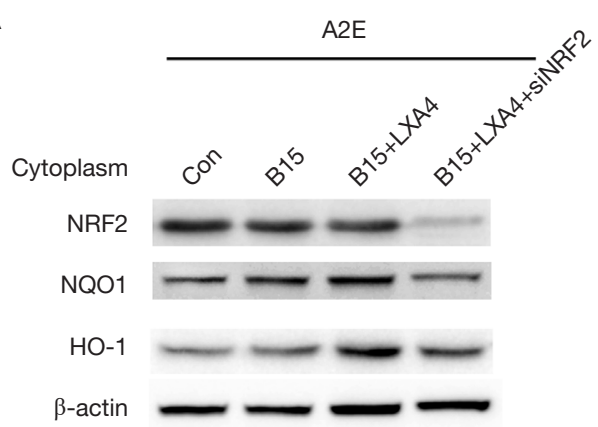

C

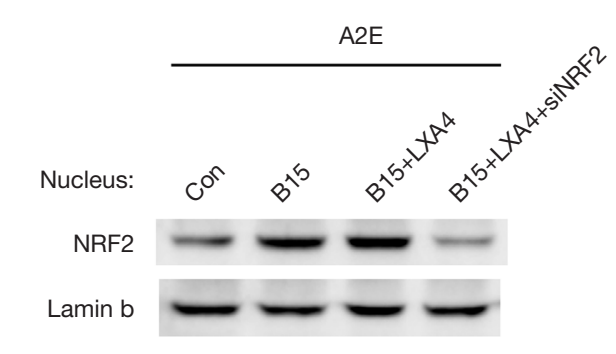

$\mathrm{F}$

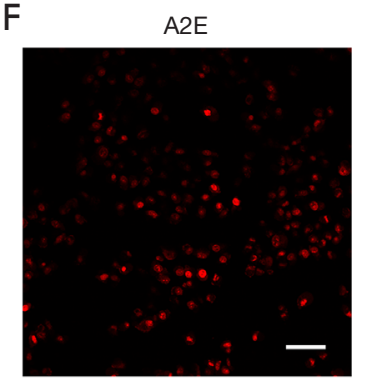

$\mathrm{A} 2 \mathrm{E}+\mathrm{B} 15+\mathrm{LXA} 4$

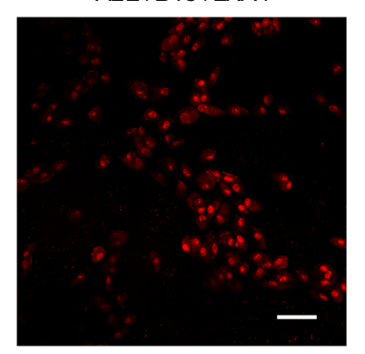

$\mathrm{A} 2 \mathrm{E}+\mathrm{B} 15$

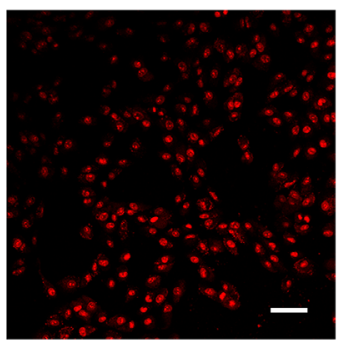

$\mathrm{A} 2 \mathrm{E}+\mathrm{B} 15+\mathrm{LXA} 4+\mathrm{SiNRF} 2$

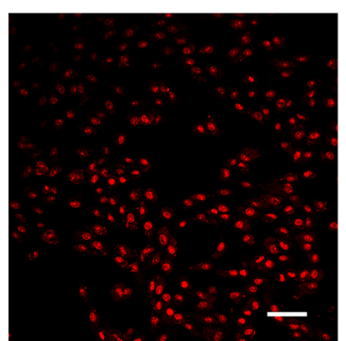

B

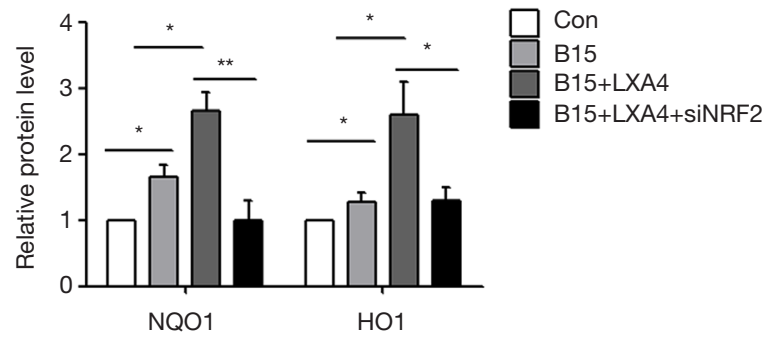

E
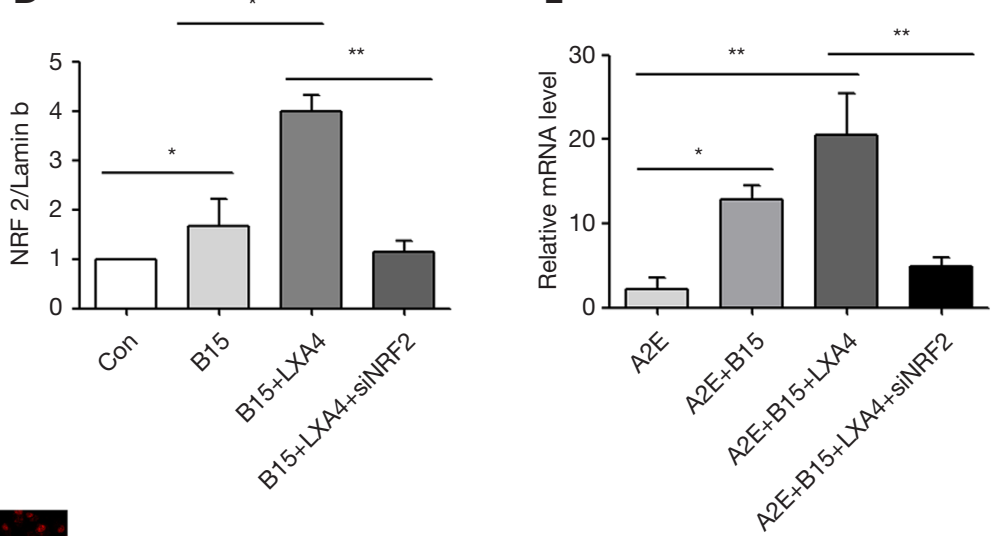

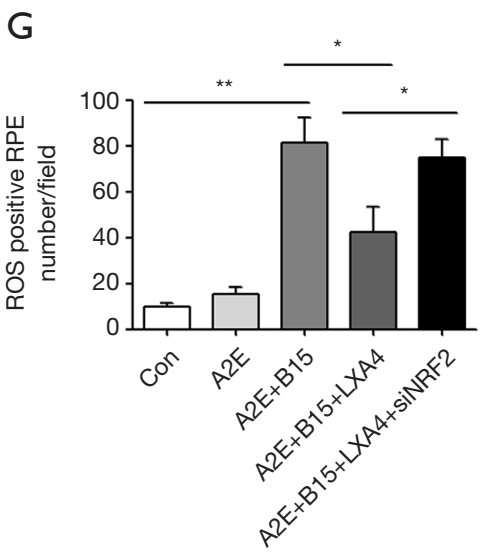

Figure 7 Blocking the NRF2-HO1 pathway reverses the protective effects of LXA4 on BL-induced ROS elimination. (A,B) The protein levels of cytoplasmic NRF2, HO1, and NQO1. Representative blots are shown, with quantification; N=4. (C,D) The levels of nuclear NRF2 were determined; $\mathrm{N}=4$. (E) The mRNA expression of HO1; N=4. (F) Representative images of DHE staining; scale bar, $50 \mu \mathrm{m}$. (G) Quantification of DHE-positive cells post BL illumination; $\mathrm{N}=4$. Con, vehicle control; A2E + B15, RPE cells treated with A2E and exposed to BL for $15 \mathrm{~h}$; A2E + B15 + LXA4, RPE cells treated with A2E and exposed to LXA4 pre-treatment for 30 min and BL for $15 \mathrm{~h}$; A2E + B15 + LXA4 + siNRF, RPE cells treated with A2E after transfected with NRF2 siRNA exposed to LXA4 pre-treatment for 30 min and BL for 15 h. The results are presented as the mean $\pm \mathrm{SEM},{ }^{*} \mathrm{P}<0.05$ and ${ }^{* *} \mathrm{P}<0.01$. RPE, retinal pigment epithelial (/epithelium) layer. 
BL-induced retinal regeneration model. Protection of LXA4 in retinal regeneration was associated with oxidative stress amelioration and antioxidant system activation. Our results also showed the Nrf2/ARE signaling pathway plays an important role in RPE cell photo-damage. Thus, LXA4 represents a potential strategy for preventing AMD development.

\section{Acknowledgments}

Funding: This work was supported by the National Natural Science Foundation of China (grant numbers: 81800845, 81770941), the Technology Development Fund (grant number: CSE12N1701), the Wuxi Taihu Lake Talent Plan, Supports for Leading Talents in Medical and Health Profession (grant numbers: 2020-THRCTD-1, THRCDJ-1) and the Top Talent Support Program for Young and Middle-Aged People of the Wuxi Health Committee (grant numbers: HB2020004, HB2020022).

\section{Footnote}

Reporting Checklist: The authors have completed the ARRIVE reporting checklist. Available at https://dx.doi. org/10.21037/atm-21-3390

Data Sharing Statement: Available at https://dx.doi. org/10.21037/atm-21-3390

Conflicts of Interest: All authors have completed the ICMJE uniform disclosure form (available at https://dx.doi. org/10.21037/atm-21-3390). The authors have no conflicts of interest to declare.

Ethical Statement: The authors are accountable for all aspects of this work, including ensuring that any questions related to the accuracy or integrity of any part of the work have been appropriately investigated and resolved. All animal experimental procedures in the present study were approved and monitored by the Institutional Animal Care and Use Committee of the Affiliated Wuxi People's Hospital of Nanjing Medical University. Animal care and method procedures were carried out in accordance with the National Institute's Health Guide for the Care and Use of Laboratory Animals (National Academy of Sciences Press, 2011).

Open Access Statement: This is an Open Access article distributed in accordance with the Creative Commons Attribution-NonCommercial-NoDerivs 4.0 International License (CC BY-NC-ND 4.0), which permits the noncommercial replication and distribution of the article with the strict proviso that no changes or edits are made and the original work is properly cited (including links to both the formal publication through the relevant DOI and the license). See: https://creativecommons.org/licenses/by-nc-nd/4.0/.

\section{References}

1. Nowak JZ. Age-related macular degeneration (AMD): pathogenesis and therapy. Pharmacol Rep 2006;58:353-63.

2. Ammar MJ, Hsu J, Chiang A, et al. Age-related macular degeneration therapy: a review. Curr Opin Ophthalmol 2020;31:215-21.

3. Sparrow JR, Zhou J, Ben-Shabat S, et al. Involvement of oxidative mechanisms in blue-light-induced damage to A2E-laden RPE. Invest Ophthalmol Vis Sci 2002;43:1222-7.

4. Lin $\mathrm{CH}, \mathrm{Wu} \mathrm{MR}$, Huang $\mathrm{WJ}$, et al. Low-Luminance Blue Light-Enhanced Phototoxicity in A2E-Laden RPE Cell Cultures and Rats. Int J Mol Sci 2019;20:1799.

5. Alaimo A, Di Santo MC, Domínguez Rubio AP, et al. Toxic effects of A2E in human ARPE-19 cells were prevented by resveratrol: a potential nutritional bioactive for age-related macular degeneration treatment. Arch Toxicol 2020;94:553-72.

6. Lee BL, Kang JH, Kim HM, et al. Polyphenol-enriched Vaccinium uliginosum L. fractions reduce retinal damage induced by blue light in A2E-laden ARPE19 cell cultures and mice. Nutr Res 2016;36:1402-14.

7. Park SI, Jang YP. The Protective Effect of Brown-, Gray-, and Blue-Tinted Lenses against Blue LED Light-Induced Cell Death in A2E-Laden Human Retinal Pigment Epithelial Cells. Ophthalmic Res 2017;57:118-24.

8. Feng J, Tan W, Li T, Yan Q, et al. Human retinal pigment epithelial cells are protected against hypoxia by BNIP3. Ann Transl Med 2020;8:1502.

9. Johnson J, Maher P, Hanneken A. The flavonoid, eriodictyol, induces long-term protection in ARPE19 cells through its effects on $\mathrm{Nrf} 2$ activation and phase 2 gene expression. Invest Ophthalmol Vis Sci 2009;50:2398-406.

10. Baird L, Yamamoto M. The Molecular Mechanisms Regulating the KEAP1-NRF2 Pathway. Mol Cell Biol 2020;40:e00099-20.

11. Kim MH, Kim DH, Yang SG, et al. Improved effect of a 
mitochondria-targeted antioxidant on hydrogen peroxideinduced oxidative stress in human retinal pigment epithelium cells. BMC Pharmacol Toxicol 2021;22:7.

12. Kim J, Cho K, Choung SY. Protective effect of Prunella vulgaris var. $\mathrm{L}$ extract against blue light induced damages in ARPE-19 cells and mouse retina. Free Radic Biol Med 2020;152:622-31.

13. Romano M, Recchia I, Recchiuti A. Lipoxin receptors. ScientificWorldJournal 2007;7:1393-412.

14. Sobrado M, Pereira MP, Ballesteros I, et al. Synthesis of lipoxin A4 by 5 -lipoxygenase mediates PPARgammadependent, neuroprotective effects of rosiglitazone in experimental stroke. J Neurosci 2009;29:3875-84.

15. Perretti M, Chiang N, La M, et al. Endogenous lipid- and peptide-derived anti-inflammatory pathways generated with glucocorticoid and aspirin treatment activate the lipoxin A4 receptor. Nat Med 2002;8:1296-302.

16. Obert E, Strauss R, Brandon C, et al. Targeting the tight junction protein, zonula occludens- 1 , with the connexin 43 mimetic peptide, $\alpha \mathrm{CT} 1$, reduces VEGF-dependent RPE pathophysiology. J Mol Med (Berl) 2017;95:535-52.

17. Yanagi Y, Inoue Y, Jang WD, et al. A2e mediated phototoxic effects of endoilluminators. Br J Ophthalmol 2006;90:229-32.

18. Qin T, Gao S. Inhibition of Proteasome Activity Upregulates IL-6 Expression in RPE Cells through the Activation of P38 MAPKs. J Ophthalmol 2018;2018:5392432.

19. Wang M, Wang Y, Xie T, et al. Prostaglandin E2/EP2 receptor signalling pathway promotes diabetic retinopathy in a rat model of diabetes. Diabetologia 2019;62:335-48.

20. Wilde C, Lakshmanan A, Patel M, et al. Prevalence of reticular pseudodrusen in newly presenting adult onset foveomacular vitelliform dystrophy. Eye (Lond) 2016;30:817-24.

21. Lee M, Li S, Sato K, et al. Interphotoreceptor RetinoidBinding Protein Mitigates Cellular Oxidative Stress and Mitochondrial Dysfunction Induced by All-trans-Retinal. Invest Ophthalmol Vis Sci 2016;57:1553-62.

22. Morris T, Stables M, Hobbs A, et al. Effects of low-dose aspirin on acute inflammatory responses in humans. J Immunol 2009;183:2089-96.

23. Shi X, Fu L. Piceatannol inhibits oxidative stress through modification of Nrf2-signaling pathway in testes and attenuates spermatogenesis and steroidogenesis in rats exposed to cadmium during adulthood. Drug Des Devel Ther 2019;13:2811-24.

24. Suzuki T, Yamamoto M. Stress-sensing mechanisms and the physiological roles of the Keap1-Nrf2 system during cellular stress. J Biol Chem 2017;292:16817-24.

25. Friedman DS, O'Colmain BJ, Muñoz B, et al. Prevalence of age-related macular degeneration in the United States. Arch Ophthalmol 2004;122:564-72.

26. Eisenhauer B, Natoli S, Liew G, et al. Lutein and Zeaxanthin-Food Sources, Bioavailability and Dietary Variety in Age-Related Macular Degeneration Protection. Nutrients 2017;9:120.

27. Arunkumar R, Calvo CM, Conrady CD, et al. What do we know about the macular pigment in AMD: the past, the present, and the future. Eye (Lond) 2018;32:992-1004.

28. Wielgus AR, Collier RJ, Martin E, et al. Blue light induced A2E oxidation in rat eyes--experimental animal model of dry AMD. Photochem Photobiol Sci 2010;9:1505-12.

29. Nakamura M, Yako T, Kuse Y, et al. Exposure to excessive blue LED light damages retinal pigment epithelium and photoreceptors of pigmented mice. Exp Eye Res 2018;177:1-11.

30. Vicente-Tejedor J, Marchena M, Ramírez L, et al. Removal of the blue component of light significantly decreases retinal damage after high intensity exposure. PLoS One 2018;13:e0194218.

31. Alaimo A, Liñares GG, Bujjamer JM, et al. Toxicity of blue led light and $\mathrm{A} 2 \mathrm{E}$ is associated to mitochondrial dynamics impairment in ARPE-19 cells: implications for age-related macular degeneration. Arch Toxicol 2019;93:1401-15.

32. Arias L, Caminal JM, Rubio M, et al. Retinal pigment epithelial tears after intravitreal bevacizumab injection for predominantly classic choroidal neovascularization. Eur J Ophthalmol 2007;17:992-5.

33. Algvere PV, Berglin L, Gouras P, et al. Transplantation of RPE in age-related macular degeneration: observations in disciform lesions and dry RPE atrophy. Graefes Arch Clin Exp Ophthalmol 1997;235:149-58.

34. Kim J, Jin HL, Jang DS, et al. Quercetin-3-O- $\alpha-1-$ arabinopyranoside protects against retinal cell death via blue light-induced damage in human RPE cells and Balb-c mice. Food Funct 2018;9:2171-83.

35. Quan JH, Ismail HAHA, Cha GH, et al. VEGF Production Is Regulated by the AKT/ERK1/2 Signaling Pathway and Controls the Proliferation of Toxoplasma gondii in ARPE-19 Cells. Front Cell Infect Microbiol 2020;10:184.

36. Cheng YS, Linetsky M, Gu X, et al. Light-induced generation and toxicity of docosahexaenoate-derived oxidation products in retinal pigmented epithelial cells. Exp Eye Res 2019;181:325-45. 
37. Wu Q, Chong L, Shao Y, et al. Lipoxin A4 reduces hyperoxia-induced lung injury in neonatal rats through PINK1 signaling pathway. Int Immunopharmacol 2019;73:414-23.

38. Wu J, Ding DH, Li QQ, et al. Lipoxin A4 Regulates Lipopolysaccharide-Induced BV2 Microglial Activation and Differentiation via the Notch Signaling Pathway. Front Cell Neurosci 2019;13:19.

39. Brennan EP, Mohan M, McClelland A, et al. Lipoxins Protect Against Inflammation in Diabetes-Associated Atherosclerosis. Diabetes 2018;67:2657-67.

40. Yang S, Zheng Y, Hou X. Lipoxin A4 restores oxidative stress-induced vascular endothelial cell injury and thrombosis-related factor expression by its receptormediated activation of Nrf2-HO-1 axis. Cell Signal 2019;60:146-53.

41. Fanjul-Moles ML, López-Riquelme GO. Relationship between Oxidative Stress, Circadian Rhythms, and AMD. Oxid Med Cell Longev 2016;2016:7420637.

Cite this article as: Xie T, Cai J, Yao Y, Sun C, Yang Q, Wu M, Xu Z, Sun X, Wang X. LXA4 protects against bluelight induced retinal degeneration in human A2E-laden RPE cells and Balb-c mice. Ann Transl Med 2021;9(15):1249. doi: 10.21037/atm-21-3390
42. Kim DH, Kim JH, Hwangbo H, et al. Spermidine Attenuates Oxidative Stress-Induced Apoptosis via Blocking Ca2 + Overload in Retinal Pigment Epithelial Cells Independently of ROS. Int J Mol Sci 2021;22:1361.

43. Zhang H, Liu YY, Jiang Q, et al. Salvianolic acid A protects RPE cells against oxidative stress through activation of Nrf2/HO-1 signaling. Free Radic Biol Med 2014;69:219-28.

44. Kapoor R, Sirohi VK, Gupta K, et al. Naringenin ameliorates progression of endometriosis by modulating Nrf2/Keap1/HO1 axis and inducing apoptosis in rats. J Nutr Biochem 2019;70:215-26.

45. Cui R, Tian L, Lu D, et al. Exendin-4 Protects Human Retinal Pigment Epithelial Cells from H2O2-Induced Oxidative Damage via Activation of NRF2 Signaling. Ophthalmic Res 2020;63:404-12.

(English Language Editor: L. Huleatt) 

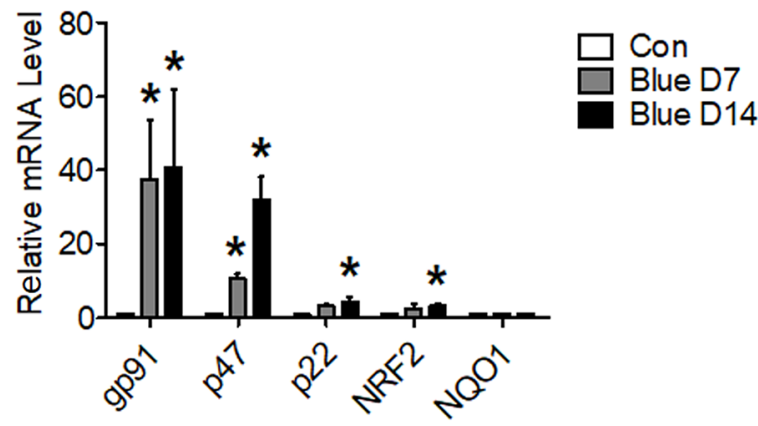

Figure S1 Oxidative stress/nitrative stress in the RPE/choroid of BL-exposed Balb-c mice. (A) Gene expression of gp91, p47 and p22 in the RPE-choroid-sclera complexes of each group was detected by real-time PCR $(\mathrm{N}=7)$. The results are presented as means \pm SEM; $\mathrm{N}=6$, ${ }^{*} \mathrm{P}<0.05$ for each pair of groups indicated.

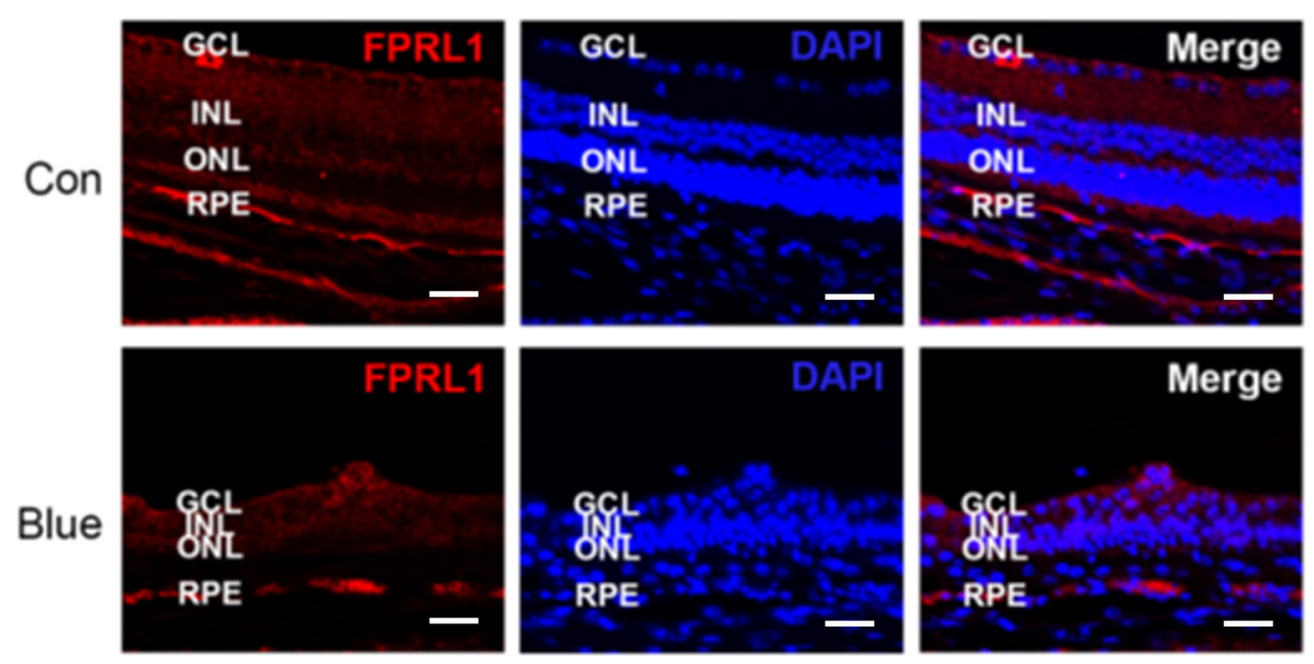

Figure S2 Expression of FPRL1 in the retina and RPE-choroid-sclera complexes post BL illumination. Representative images of immunostaining of FPRL1 in the retina and RPE-choroid-sclera complexes of each group. Scale bar =25 $\mu \mathrm{m}$. GCL, ganglion cell layer; INL, inner nuclear layer; ONL, outer nuclear layer; RPE, retinal pigment epithelial (/epithelium) layer. 


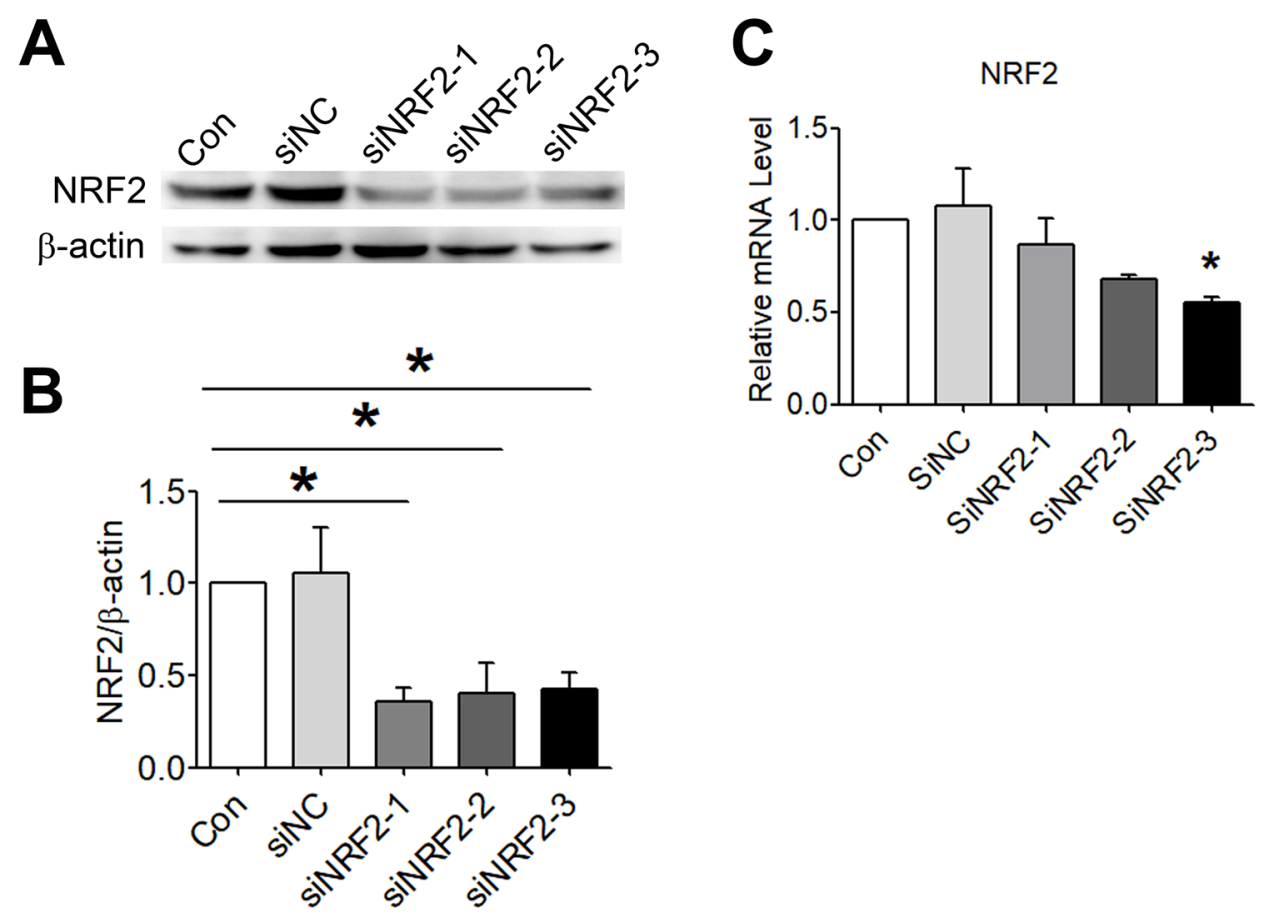

Figure S3 The level of NRF2 in hRMECs after siRNAs transfection. (A,B) Cells were transiently transfected with the expression vector encoding NRF2 siRNAs or the empty vector. Lysates of the cells were immunoblotted using the anti-NRF2 antibody; N=5. (C) Gene expression of NRF2 in each group's RPE cells was detected via real-time PCR; $\mathrm{N}=5$. The results are presented as means $\pm \mathrm{SEM}$; ${ }^{\mathrm{P}}<0.05$ for each pair of groups indicated.

Table S1 Primer sequences and conditions for conventional RT-PCR

\begin{tabular}{lll}
\hline Genes & Forward primer (5'-3') & Reverse primer (5'-3') \\
\hline Human NRF2 & GGTGATGATTTTACTCTGC & TTTCCGAGTCACTGATGAACC \\
Human HO1 & TCTTGGCTGGCTTCCTTAC & CATAGGCTCCTTCCTCCTTTC \\
Human GAPDH & ATCACCATCTTCCAGGAGCG & CGCCTGCTTCACCACCTTCTTG \\
Mouse NRF2 & TCACACGAGATGAGCTTAGGGCAA & TACAGTTCTGGGCGGCGACTTTAT \\
Mouse NQO1 & TGGCCGAACACAAGAAGCTG & GCTACGAGCACTCTCTCAAACC \\
Mouse gp91 & AGCTATGAGGTGGTGATGTTAGTGG & CACAATATTGTACCAGACAGACTTGAG \\
Mouse p22 & CTGCCCTCCACTTCCTGTTG & TTCACCCTCACTCGGCTTCT \\
Mouse p47 & ATGAAGGTCTCCACCACTG & GCATTCAGTTCCAGGTCA \\
Mouse-HRPT & CCCCAGGAATACAAAGACT & AACAAGAGCATAGCAGGAAC \\
\hline
\end{tabular}

